\title{
L'œuvre de Kim Thúy: une écriture née de la fêlure biographique
}

\section{Kim Thúy's work: a writing born of the biographical crack}

\author{
Ángeles SÁnchez Hernández \\ Universidad de Las Palmas de Gran Canaria \\ angeles.sanchez@ulpgc.es
}

\begin{abstract}
Resumen
En este artículo analizamos la obra de Kim Thúy, escritora canadiense de origen vietnamita. Sus textos se asemejan al género autobiográfico porque las historias que cuenta siguen de cerca su trayectoria biográfica y están escritos en primera persona; sin embargo, ciertos elementos no coinciden con el recorrido íntimo de la autora ni con los rasgos de la formulación general del género; por lo tanto, no se trata de una autobiografía normativa. Revisamos las teorías actuales sobre autobiografía y autoficción como fundamentación para nuestro análisis. A través de sus libros, estudiamos el lado de las escrituras del yo que se desvía de la idea tradicional de la autobiografía exponiendo una evolución de este tipo de relato relacionada con los cambios sociales del siglo XXI.
\end{abstract}

\section{Palabras clave}

Kim Thúy, autobiografía, autoficción, hibridismo.

\begin{abstract}
In this article we analyse the work of Kim Thúy, a Canadian authoress of Vietnamese origin. Her texts resemble the autobiographical genre because the stories she tells closely follow her biographical trajectory and are written in first person; yet some elements do not coincide with the author's intimate journey or with the features of the genre's general formulation; therefore, it is not a normative autobiography. We review the current theories of autobiography and auto-fiction as the basis for our analysis. Through her books, we study the autobiographical works that deviates from the traditional idea of autobiography by exposing an evolution of this type of story related to the social changes of the 21 st century.
\end{abstract}

\section{Kay-words}

Kim Thúy, autobiography, auto-fiction, hybridism. 


\section{Introduction}

Dans cet article, nous proposons d'analyser l'œuvre de Kim Thúy, écrivaine canadienne d'origine vietnamienne. D'après nous, elle offre une approche singulière aux écritures de soi, présentant une thématique qui aborde des questions brûlantes du $\mathrm{XXI}^{\mathrm{e}}$ siècle en tant qu'être hybride appartenant à deux cultures. La narratrice se dévoile par à-coup, revenant sur un détail du passé de son enfance vietnamienne pour le relier au présent sur la terre canadienne. La singularité de son œuvre tient à la façon de raconter des événements intimes et sociaux par la précision des mots et par la structuration des récits qui mettent en valeur la dichotomie identitaire et les évènements vécus sous un ton de légèreté joyeuse.

D'emblée, ses textes s'approchent du genre autobiographique car les fictions sont collées à la trajectoire vitale de l'auteure comme l'on peut facilement constater même si les prénoms des narratrices ne coïncident pas avec celui de l'écrivaine; donc, il ne s'agit pas d'une autobiographie normative. En revanche, il y a toujours ce "je” au féminin, né au Vietnam à un moment précis du passé de ce pays, pour raconter son histoire rétrospectivement. La première personne établit des passerelles incontournables avec la vie passée et actuelle de Thúy; les éléments de l'histoire se raccordent bien à sa biographie. Le lecteur se demande si, par exemple, ru est une autobiographie ou, simplement, un récit composé avec des éléments vécus par la narratrice pour composer sa fiction. Nous souhaitons approfondir l'étude du versant des écritures du moi qui ne colle pas à l'idée traditionnelle d'autobiographie et qui formule une évolution de ce genre, liée aux changements sociaux et culturels du XXI ${ }^{\mathrm{e}}$ siècle.

Alors, pour mener notre analyse, nous tenterons de délimiter, en tant qu'il se peut, les contours théoriques entre autobiographie et autofiction, pour préciser ensuite le sens de la démarche scripturale soutenue par Kim Thúy. Puis nous retracerons brièvement quelques données de la biographie de Thúy pour décrire après notre corpus d'étude composé de trois publications: $r u, m a \tilde{n}$ et $v i^{1}$, en faisant ressortir les éléments thématiques et stylistiques qui distinguent l'œuvre de cette écrivaine. Notre hypothèse d'étude part d'une impression en tant que lecteur concerné par ses romans où tout semble simple et léger et, cependant, sa lecture nous imprègne des questions bouleversantes de la problématique existentielle. Nous tenterons de conclure, à la suite de cette analyse, où réside la singularité de l'œuvre de cette écrivaine par rapport aux écritures du moi.

\section{L'enjeu de l'autobiographie et de l'autofiction dans l'écriture}

Les formes qu'adoptent les écritures de soi sont multiples. Pierre Brunel (2008: 10) réfléchit sur l'écriture autobiographique et admet qu'on devrait pouvoir fixer deux limites précises; d'un côté, celui de la fiction pure sans aucun élément suspect de procéder d'une

1 Les titres apparaissent en minuscule sur la couverture des livres. 
autobiographie déguisée de l'auteur et, d'un autre, l'autobiographie pure sans aucun détail inventé. Mais, étant donné la difficulté de retrouver cette pureté narrative, ce chercheur constate que la masse même de la littérature évolue entre les deux et pas seulement celle de la littérature romanesque.

Les études de Philippe Lejeune sur l'autobiographie ont marqué définitivement la vision théorique sur le sujet. Il définit ce genre comme un "récit rétrospectif en prose qu'une personne réelle fait de sa propre existence, lorsqu'elle met l'accent sur sa vie individuelle, en particulier sur l'histoire de sa personnalité" (Lejeune, 1975: 14). Cette définition implique plusieurs composantes; d'abord, la forme du langage qui présuppose un récit en prose, puis il faut un sujet racontant une existence où l'identité de l'auteur et du narrateur coïncident, de même que l'identité de narrateur et du personnage principal; et, enfin, l'histoire est racontée d'un point de vue rétrospectif. Dans les genres voisins comme la biographie, le roman personnel et le poème autobiographique, toutes ces conditions ne sont pas remplies; cependant, il y a une condition tout à fait indispensable à ces types de récit: l'identité. Il faut absolument que l'auteur, le narrateur et le personnage principal soient la même personne et que cette identité se manifeste dans le texte par l'emploi de la première personne: je. On peut aussi utiliser la troisième "il/elle" ou même la seconde "tu", mais c'est plus rare car, comme nous rappelle Zanone (1996: 12): "L'autobiographie exprimée à la troisième personne est possible, mais à condition d'un habillage habile du narrateur, afin de cacher son lien d'identité avec le personnage principal". Le récit autobiographique fait l'histoire de la personnalité du narrateur, mais il ne s'agit pas simplement de raconter des événements passés car, très souvent, on effectue aussi une évaluation constante à l'instar du héros d'un roman d'apprentissage pour qui chaque péripétie est le moment d'une formation et une occasion de mûrissement à retenir.

Le récit autobiographique a souffert des métamorphoses "depuis quelques années et les notions critiques tournent autour des tentatives littéraires multiples qui se jouent sur la scène complexe du sujet, aux feux chatoyants de l'écriture" selon Bruno Blanckeman (2002: 111). Ces mutations ont produit une prolifération des termes pour désigner cette modalité d'écriture: autofabulation, autonarration, fictionnalisation de soi, ou autofiction (Le Quellec Cottier, 2012: 21). Selon Damien Zanone (1996: 14), “L'enjeu de l'autobiographie est d'établir, malgré l'action dissolvante du temps, l'unité de son individualité". Cette unité identitaire se perçoit dans les ouvrages de Kim Thúy, sa personnalité est construite à travers le temps et les événements vécus, c'est une individualité autre, issue de la fusion de deux cultures éloignées. L'écriture de cette auteure diverge en bien des points de l'autobiographie 'normative' et nous voulons déterminer en quoi elle s'oppose et en quoi elle se plie aux règles des écritures où le sujet-auteure est au centre de l'histoire.

Les recherches de Gasparini (2011: 11) élargissent le discours critique des écritures du moi soutenant que "le récit autobiographique développe une réflexion critique sur la genèse du sujet, sur son identité, sur sa précarité, sur ses mutations. [...] elle peut aussi se 
tourner vers les autres [...] dans une perspective axiologique". Cet aspect identitaire du sujet et du regard que celui-ci porte sur les autres nous intéresse particulièrement pour l'analyse de l'œuvre de Thúy car nous partageons l'avis d'Isabelle Grell (2014: 108) quant à la raison d'être de la littérature qui est "un rapport passionné à la vérité insaisissable du monde. Ôtez l'homme, les histoires ne sont plus". Les histoires racontées par cette auteure étalent l'aventure d'un être humain en constante interaction avec le monde et son entourage. Dans ses récits, la problématique identitaire est fondamentale, mais il ne faut pas oublier l'intérêt pour ceux qui sont concernés par ce même questionnement. Elle dépasse le moi egocentrique pour aller à l'encontre des 'sans voix', ceux qui sont incapables de s'inscrire dans l'Histoire ou dans l'imaginaire collectif parce qu'ils n'ont pas de parole publique pour se faire entendre. L'écriture littéraire leur procure cette inscription dans l'imaginaire à travers la voix et le témoignage des écrivains qui ont partagé leurs expériences.

Les écritures du moi doivent restituer la réalité "qui est imprimée en nous [vous]" selon Grell (2014: 35-36), elle constate que les failles d'où surgissent les textes n'ont rien de ce que Philippe Forest appelle "faux témoignage". Par contre, en analysant plusieurs types d'écritures autofictionnelles, elle prend en exemple le travail de Camille Lauren où elle observe que les faits énoncés sont vrais suivant l'idée du "livre intérieur". Les récits autofictionnels peuvent être déplacés ou recomposés, mais ils permettent de s'interroger sur la mémoire, l'oubli, l'effacement ou le temps. La démarche de quête de la vérité de Laurens est différente à celle d'Annie Ernaux, car cette écrivaine normande refuse toute possibilité de fiction; elle est obsédée par le vrai et elle rétablit les instants vécus par des descriptions minutieuses, partant souvent d'une photo. C'est pour cette raison qu'elle revient dans ses ethnotextes sur un même instant pour établir des perspectives multiples sur un même fait car elles reconstruisent mieux le vrai moment vécu.

Étant donné la variabilité d'écritures autobiographiques, il faut se poser la question de l'implication de la mémoire et de l'imagination dans toutes les modalités littéraires où le moi de l'auteur est au centre de la narration car toutes les deux sont liées. Philippe Lejeune (2010: 21) confirmait déjà que “nous devons garder à l'esprit que l'autobiographie n'est qu'une fiction produite dans des conditions particulières" parce que, dès que les faits entrent dans l'espace scriptural, l'auteur-narrateur va interpréter les événements vécus depuis l'expérience d'un être autre, différent à celui qui subit le fait relaté. C'est-à-dire, ce qu'on préjuge comme vrai est enjolivé ou affadi par la mémoire et l'imagination; souvent, l'imagination tente de remplir les trous de mémoire qui surgissent indéfectiblement pour réaliser le but d'esthétiser le texte. Ni l'autobiographie ni l'autofiction ne peuvent relater la vérité intacte et originale de leur vécu car elles présentent une vérité remaniée et reconstituée. "L'autobiographe promet de dire toute la vérité alors que l'autofictionneur rappelle au contraire qu'il ne peut pas tout dire mais promet que ce qu'il relate est vrai” (Tavana, Nassehi \& Azghandi, 2013: 138).

Jusqu'ici nous avons essayé de réviser les recherches sur l'autobiographie et l'autofic- 
tion qui sont la clé pour conduire notre étude; mais il faut, de plus, se poser une autre question par rapport à cette investigation, celle de la fonction que remplissent ces écritures du moi dans la littérature. Elena Cuasante (2018: 27-29) a mis au point une synthèse de diverses motivations et des fonctionnalités de cette typologie textuelle. Elle distingue trois types de motivations: les motivations rationnelles qui avancent du domaine du moi privé au moi social; les motivations affectives où l'écriture suppose, d'une certaine manière, un refuge; et, enfin, les motivations hybrides pour le simple plaisir d'écrire. Dans le premier type de motivations, elle souligne un aspect que nous trouvons essentiel, celui qui parle de la dimension existentielle qui apparaît dès le moment même où l'individu prend la décision consciente d'écrire. Elle repère ensuite une autre perspective par rapport à la fonction des écritures autobiographiques, celle de la transcription textuelle de faits pour mettre en ordre ce qui a été vécu, mais ce procès va plus loin de la simple récapitulation des événements puisqu'il suppose aussi une interprétation des actes. Des fonctions notées par Cuasante Fernández, nous retenons, de plus, la fonction gnoséologique pour notre analyse car elle est reconnue comme essentielle par les critiques qui étudient les littératures du moi. Cette dernière fonction découle de la dimension existentielle, du besoin de connaissance de l'être humain, car écrire sur soi-même est une manière de réagir au besoin de comprendre l'existence sans quitter sa propre sphère, elle donne la possibilité d'avoir une compréhension approfondie de soi-même et de son entourage. L'action d'enregistrer en mots ce que l'auteur a vécu constitue une manière indéniable de mettre en ordre l'expérience de la formation identitaire parce que "l'écriture autobiographique est moins un processus de récapitulation que d'interprétation", selon Cuasante Fernández (2018: 28).

Depuis le dernier quart du siècle passé, l'écriture des femmes et celle de tous ceux qui se perçoivent dans l'entre-deux — des individus provenant souvent des anciennes colonies, des exilés ou de ceux qui ont du mal à se situer dans la société- a trouvé dans les variations des écritures du moi un champ particulier pour s'exprimer, pour se dire. Ces auteurs, "poussés par le contexte général de renversement des cadres prédéterminés de la pensée, osent se débarrasser des lourdes traditions sans pour autant s'enfermer dans un camp" (Grell, 2014: 70). Il n'y a pas de critères formels pour distinguer les genres factuels des genres fictifs, il n'y a pas non plus de différences fonctionnelles pour résoudre cette tâche (Cuasante Fernández, 2018: 37). Lorsqu'il s'agit de textes invariablement qualifiés de littéraires, la fonction ludique et surtout la fonction esthétique — très sous-développée en général dans le domaine non-fictionnel — jouent un rôle prépondérant car l'écriture de soi est résolument inventive; fictive ou réelle, cette écriture, engageant l'auteur ou le lecteur, "semble procéder d'une expérience ontologique" (Gouiffès, 2002: 47). Cette manière d'écrire suppose la prise de conscience de la non-coïncidence de soi à soi; c'est-à-dire, elle surgit du sentiment d'un manque qui appelle à être comblé par le biais de l'écriture.

Pour d'autres critiques, "l'écriture autofictionnelle nait d'un besoin thérapeutique" 
(Thériault, 2016: 28). L'autofiction constitue un acte de "partage (cathartique?) de tranches de vie, de morceaux de chair, un acte qui montre, démontre, démonte l'idée d'un MOI uni, unique" (Grell, 2016: 9). Nous partageons l'idée d'écriture fictionnelle comme un acte d'interprétation du passé et du présent pour réunifier une identité dépecée qui aboutit à se comprendre par des faits constatés mais qui réussit à se recomposer par des fictions qui comblent les trous de mémoire avec les expériences de son entourage. L'écrivain suisse Paul Nizon, parlant du but de son travail littéraire, affirme qu'il n'est pas intéressé à la reconstruction du passé mais plutôt qu'il s'intéresse au moi en tant que composante changeante et insaisissable de l'être humain. Il avoue qu'“il s'agit, en écrivant, de descendre vers ce moi inconnu afin de le constituer d'une manière ou d'une autre, comme personnage. Le 'je' n'est donc pas le point de départ, comme dans l'autobiographie, mais le point d'arrivée" (Nizon, 2005: 126).

L'œuvre de Thúy est à la recherche de ce moi qui englobe le passé et le présent de la narratrice, mais ce moi tient compte de la société qui entoure ces personnages partagés entre deux cultures. En ce sens, l'œuvre de l'écrivaine québécois-vietnamienne tisse des passerelles avec d'autres écrivaines francophones qui s'occupent de ceux dont la voix a été passée sous silence par l'Histoire. L'écrivaine ${ }^{2}$ admet dans un entretien télévisé qu'il peut s'agir d'une autofiction mais qu'elle voudrait que beaucoup de Vietnamiens qui ont vécu l'exode puissent se retrouver dans cette histoire. Les histoires des boat people n'ont pas été beaucoup racontées dans la littérature, le but de l'auteure est de les inscrire par ses mots. Elle reconstruit son histoire et celle de ses proches à partir de "bulles de mémoire" (Chaudey, 2010), et elle ne manifeste pas d'intérêt personnel à s'inscrire dans aucun genre précis.

\section{Bref aperçu de la vie et l'œuvre de Kim Thúy}

Kim Thúy est née en 1968 à Saigon au Vietnam "pendant l'offensive du Têt, aux premiers jours de la nouvelle année du Singe, lorsque les longues chaînes de pétards accrochées devant les maisons explosaient en polyphonie avec le son des mitraillettes" (Thúy, 2009: 11). Malgré la guerre, elle a passé son enfance dans l'aisance mais, à 10 ans, elle a fui la dictature communiste et a quitté le Vietnam avec sa famille sur un bateau qui l'a conduite vers le Canada. Après un séjour dans un camp de réfugiés en Malaisie, ils arrivent à Granby au Québec où leur vie va absolument changer. Le père de Kim Thuy, philosophe de formation, avait été député dans son pays d'origine mais, arrivé au Canada, il doit livrer des pizzas ou tenir la caisse d'un dépanneur pour subsister. Sa mère, dont le grand-père avait été préfet au Vietnam, est obligée de faire le ménage des maisons ou de coudre à la machine, aidée par ses enfants. La future écrivaine découvre à 15 ans la littérature grâce à l'Amant, le récit des amours vietnamiens de Marguerite Duras. Ce livre a été une révélation pour elle qui avait déjà le goût des mots depuis petite. Elle travaille pour se payer les études, elle fera finalement du Droit

2 Entretien télévisé dans l'émission 'Tout le monde en parle', 2010, disponible sur <https://www.youtube.com/ watch?v=Ft3XXKAA1zw> 
et devient avocate dans l'un des plus importants cabinets internationaux de Montréal avant d'être envoyée en mission à Hanoi. Thúy explique qu'il lui a fallu tout réapprendre du Viêtnam mais, finalement, elle exprime son attachement et son amour au pays natal (Chaudey, 2010). Les histoires de ses romans sont suspendues entre passé et futur et la transmission familiale y occupe une place de choix.

En 1998, elle retourne au Vietnam envoyée en mission commerciale par son cabinet d'avocats; elle restera trois ans à Hanoi où son mari l'a rejointe. C'est en Asie où Kim Thuy va alors vivre plusieurs années et mettre au monde ses deux enfants. En 2007, après la fermeture des portes de son restaurant à Montréal 'Ru de Nam', elle s'accorde un moment de détente où elle se voue à l'écriture. Kim Thuy avait gardé en mémoire l'histoire d'un vieil immigré à qui l'écriture avait permis de sauver sa peau dans les camps de prisonniers (Chaudey, 2010). L'année 2009 marque alors un autre tournant de sa vie puisque paraît son premier roman: $r u$. Très vite le succès de l'ouvrage est retentissant, elle se voit louée par les critiques française et québécoise, recevant de nombreuses récompenses.

Notre travail se focalise sur les trois textes qui racontent des histoires pliées à son expérience personnelle: ru (2009), mãn (2013), vi (2016), et nous laissons de côté le livre des échanges épistolaires qu'elle publie en 2011, À toi, cosigné avec Pascal Janovjak, l'écrivain franco-suisse qui habitait à l'époque de la rédaction à Ramallah, en Palestine. Les deux écrivains, aussi passionnés l'un que l'autre par l'écriture, vont partager leurs réflexions et leurs inquiétudes, parlant du quotidien et des souvenirs avec beaucoup d'humour et sensibilité. Dans le premier roman, $r u$, l'auteure raconte son itinéraire et celui d'autres réfugiés vietnamiens. La signification du titre 'petit ruisseau' en français est complémentaire de l'acception en vietnamien 'berceuse'. Elle restitue par l'écriture les faits du passé vietnamien et son parcours personnel en miroir du devenir de tant d'autres compatriotes qui ont fui leur pays; ce livre reste peut-être le plus près de sa biographie personnelle. Son deuxième roman, mãn, détaille, d'après le témoignage de Thúy, 'l'apprentissage du mot 'aimer' pour donner suite à la définition du verbe 'vivre' de $\grave{A}$ toi et à la conjugaison de 'survivre' de $R u$ " (Nancy, 2013) 3 . Ce récit semble, à première vue, le plus éloigné de sa biographie et, donc, la fiction y semble prendre plus de place, mais l'histoire raconte toujours l'existence d'une immigrante vietnamienne arrivée au Québec à l'âge adulte après son mariage; le récit pivote sur une restauratrice qui réussit à s'exprimer et à s'intégrer par la cuisine, on constate que cette expérience de cuisinière n'est pas éloignée non plus du parcours professionnel de Thúy. Et le dernier livre en publication, $v i$, titre adapté du prénom de la narratrice, Bao Vi, relate l'histoire du parcours d'apprentissage d'une jeune immigrée qui retrouve son pays d'enfance étant déjà une jeune avocate. Cette femme essaie de maintenir les préceptes de la culture vietnamienne qui oblige à suivre la chaîne familiale pour se construire. Les Vietnamiens pensent que nous ne sommes que le résultat de nos ancêtres. La brièveté des titres coïncide avec la simplicité apparente de

3 Article sur Internet sans page foliotée référé en bibliographie. 
sa narration et la sobriété de ses mots; l'écrivaine reconnaît une prédilection "par l'épuration de la phrase, pour faire en sorte qu'il ne reste que l'essentiel, que les images" (Dussaillant Fernandes, 2010: 165).

La question de la double appartenance constitue un problème essentiel dans l'œuvre de Kim Thúy qui est consciente d'embrasser les deux identités qui sont en elle sans conflit intime (Chauday, 2010). Ses récits font écho de cette perspective détendue sans antagonisme entre les deux cultures. Elle envisage son identité sans négliger ni l'examen intime ni l'engagement social. Les deux langues de l'auteure sont présentes dans ses textes, bien que la prééminence du français soit incontestable, néanmoins les traces de la langue vietnamienne sont toujours inscrites d'une manière ou autre. Elle rédige ses romans en français car c'est la langue de ses émotions, celle qui unifie ses voix intérieures et dans laquelle elle a saisi de façon plus réfléchie la réalité du monde, mais elle inscrit des traces de sa langue maternelle et même de l'anglais dans ses textes.

\section{Le je polyédrique de Kim Thúy dans trois romans: $r u$, mãn et vi}

Suivant les présupposés qu'on vient de développer dans le cadre théorique, nous allons maintenant faire l'analyse des trois romans de Thúy où l'auteure narre des histoires qui sont collées à son parcours biographique. Les narratrices qui y assurent le récit de l'histoire mènent une réflexion critique sur la genèse de leur identité et de leurs mutations à travers le temps et les interactions avec la société pour mettre en évidence les valeurs qui les ont nourries dans cette perspective axiologique notée par Gasparini que nous avons citée ci-dessus. D'ailleurs, cette perspective rejoint la fonction gnoséologique notée par Causante Fernández qui est reconnue comme essentielle dans la littérature du moi parce que le besoin d'écrire surgit souvent comme réponse au besoin de comprendre l'existence sans quitter sa propre sphère, celle où l'expérience du 'je' narrateur s'appuie sur l'expérience existentielle de l'auteur. Kim Thuy ${ }^{4}$ réaffirme le fait d'écrire comme une nécessité et explique le pouvoir de la littérature pour retisser des liens avec des mondes perdus.

\section{1. $r u$}

Ce livre lui a permis de "trouver enfin l'équilibre et la paix" d'après ses propres mots (Chaudey, 2010). La dédicace du livre, “aux gens du pays", nous laisse un peu dans l'attente de savoir de quel pays parle-t-elle. Ensuite, le lecteur est intrigué à l'ouverture du livre par les courts chapitres qui n'arrivent pas à remplir une demi-page; de toute façon, nous ne pouvons pas dire que ce sont des chapitres car il n'y a pas de titres ni de marques de séparations sauf les espaces en blanc; la narration se poursuit avec le point de liaison de la voix narratrice,

4 Vidéo en ligne proposée par la Libre Belgique publiée le dimanche 12 mars 2017. 
An Thinh. Nous apprenons, dès l'incipit, le lieu où elle est venue au monde et dans quelles conditions. Ce 'je' qui raconte nous apprend le parcours de sa vie à travers une écriture fragmentaire axée sur des 'bulles' de mémoire comme Thúy aime les nommer. Cette structure narrative devient un attrait pour poursuivre la lecture. Elle expose la vie d'avant à Saigon entrelacée à celle du Québec, l'Orient éclairant l'Occident, ou l'inverse. Cette écriture fragmentaire a été étudiée par Johanne Lalonde (2018: 35) qui souligne que ce procédé "évoque la cristallisation d'une personne au temps présent avec ses passé et futur soudés presque sans distinction". C'est la prise de conscience d'une personnalité adulte du chemin traversé pour aboutir à une identité où deux parties sont assemblées.

Dès la deuxième page, Nguyen An Thinh relie son destin à celui de sa mère, suivant ainsi la tradition vietnamienne qui avait déjà marqué cette prédestination sur le prénom de la petite fille, leurs prénoms ne divergent que d'un point sous l'i. De plus, la figure de la mère acquiert un double sens dès le début; d'un côté, la narratrice était destinée à rester la prolongation de sa mère dès sa naissance et, d'un autre côté, l'écrivaine ainsi que An Thinh sont mères de deux enfants dont l'un d'eux est autiste. Ce handicap lui a appris une autre façon d'agir dans la vie et d'établir une communication qui va plus loin des mots. Alors, la narration regarde en deux directions: vers le passé de la mère et la tradition vietnamienne et vers l'avenir et l'éducation des enfants, deux univers où les règles du jeu sont bien distinctes. Le récit évolue au fil de l'histoire chronologique, de son pays d'origine à son histoire actuelle au Canada en tant que mère chargée de transmettre des pans de la tradition à ses enfants et de découvrir au lecteur le quotidien de ceux qui ont dû fuir le pays sous l'invasion communiste. Le récit se fait par cette femme qui est guérie des blessures du passé s'il y en avait eu et qui a "rejoint son [mon] affection à Hanoi” (Thúy, 2009: 43) après avoir passé trois ans au Vietnam. Malgré l'absence de chapitres dans le livre, la progression devient logique par un subtil fil conducteur ciblé qui passe du souvenir à l'événement actuel, du passé au présent et du Vietnam au Canada où l'évocation d'un acte nous amène illico à un autre en cohésion thématique.

Après la publication de $r u$, Kim Thúy avoue prendre l'excuse de raconter 'à travers elle' l'histoire de tous ces gens qu'elle a croisés (Fortin, 2009). Elle constitue le maillon nécessaire de la chaîne de transmission de cette histoire comme corrobore son personnage: "Je raconte ces anecdotes à Pascal pour garder en mémoire un pan d'histoire qui ne trouvera jamais sa place sur les bancs de l'école" (Thúy, 2009: 46). La narratrice — comme Thúyéprouve le besoin de raconter les événements de la vie quotidienne des gens simples qui ne sont pas inscrits dans l'Histoire. Elle reconstruit le vécu d'elle-même et de tant d'autres exilés vietnamiens qui ont partagé les mêmes expériences. Par conséquence, l'écriture depuis la première personne lui confère le sceau de vérité, de témoignage des faits authentiques observés autour d'elle. Elle expose la situation de son pays natal séparé par un mur idéologique mettant en avant un mur précis, celui construit chez elle, entre sa famille et les envahisseurs, "érigé pour établir deux adresses: une pour nous et une pour le poste de police du quartier" 
(Thúy, 2009: 38). Cette cloison devient un vrai parapet de séparation entre deux univers, entre la vie rêvée de l'enfance et le présent difficile de l'époque communiste qui montre le point de départ d'une autre vie insoupçonnée où toutes les craintes avaient leur place.

La narratrice revient au Vietnam "pour travailler avec ceux qui sont à l'origine de ce mur, qui ont imaginé cet outil pour briser ces centaines de milliers de vies, peut-être des millions" (Thúy, 2009: 42). Le retour au pays d'origine en tant que femme adulte lui a fait oublier sa rancune contre "ceux qui furent le bras droit et le bras gauche de Hô Chí Minh" (Thúy, 2009: 44). Après avoir dépassé ce conflit intime, elle souhaite donner la voix aux femmes vietnamiennes car personne ne connait leur souffrance. Les hommes ont porté les armes pour lutter ou ont été emprisonnés dans les camps de rééducation et, souvent, ils y sont morts; ces événements sont connus de tous par les médias; par contre, “on oublie souvent l'existence de toutes ces femmes qui ont porté le Vietnam sur leur dos pendant que leur mari et leurs fils portaient les armes sur le leur" (Thúy, 2009: 47). Ces femmes qui traversaient le pays en train vers les camps de rééducation où étaient confinés leurs proches, elles y allaient sans aucune certitude de les retrouver vivants pour leur apporter quelques ravitaillements. La cuisine sert de liaison entre passé et présent car la narratrice maintient, sur le pays québécois, l'habitude de cuisiner les plats de viande rissolée de ces femmes-là, en souvenir d'elles. La mémoire des faits vécus quotidiennement doit se maintenir à travers le récit littéraire pour que l'exploit de ces hommes et femmes, insignifiants socialement, soit retenu dans l'imaginaire collectif. Ce sont des vies 'minuscules' comme celles figurées par Pierre Michon ou comme les personnages d'Annie Ernaux; par l'écriture de ces écrivains, leurs personnages, symboles des hommes humbles menant des vies communes, sont inscrits dans l'Histoire.

En dépit de la difficulté de vivre au temps de l'occupation du sud du Vietnam, le récit de l'histoire se fait sous un ton d'humour comme dans la scène où ces soldats, faisant inventaire des propriétés, confondent toute une armoire où était rangée la lingerie fine importée de Paris pour les femmes de la famille avec des filtres à café. Elle écrit à propos d'un soldat: "il ne connaissait pas l'utilité de ces soutiens-gorge. Selon lui, ils ressemblaient aux filtres de café de sa mère, faits de tissu cousu autour d'un anneau en métal dont un bout torsadé servait de manche" (Thúy, 2009: 39). La pauvreté était leur caractéristique essentielle, pénuries matérielles mais carences culturelles surtout. Le travail comme avocate pendant trois ans à Hanoï lui a fait comprendre et prendre du recul dans ses jugements pour reconsidérer les attitudes des soldats communistes qui avaient envahi la maison. La légèreté du jugement et la bienveillance du ton narratif siège sur la compréhension de l'ignorance des envahisseurs et leur incapacité à se soulever contre le pouvoir communiste. Ce roman rend hommage à ses parents qui ont perdu leurs richesses car ils n'ont pu sauvegarder les biens qui leur appartenaient, mais elle est fière de son héritage qui dépasse le patrimoine tangible. À propos des parents, elle note "qu'ils nous ont déjà légué la richesse de leur mémoire qui nous permet de saisir la beauté d'une grappe de glycine, la fragilité d'un mot, la force de l'émerveillement. Plus 
encore ils nous ont offert des pieds pour marcher jusqu'à nos rêves, jusqu'à l'infini” (Thúy, 2009: 50). Reconnaissance du patrimoine culturel et de la force d'esprit offerts par leurs ancêtres qui leur ont permis d'apprécier des aspects de la vie plus bénéfiques que l'argent.

$R u$ devient presque un acte d'exorcisme du passé révolu, c'est le bilan d'une vie qui apprécie le passé qui l'a traquée inconsciemment pour se libérer de ce poids colporté silencieusement. Les mots du roman se dirigent aux lecteurs pour leur dire que toute l'histoire ne prend de sens qu'avec leur lecture. Elle écrit dans la dernière page (Thúy, 2009: 142):

\begin{abstract}
Seuls autant qu'ensemble, tous ces personnages de mon passé ont secoué la crasse accumulée sur leur dos afin de déployer leurs ailes au plumage rouge et or, avant de s'élancer vers le grand espace bleu, décorant ainsi le cil de mes enfants, leur dévoilant qu'un horizon en cache toujours un autre [...] quant à moi, il en est ainsi jusqu'à la possibilité de ce livre, jusqu'à cet instant où mes mots glissent sur la courbe de vos lèvres, jusqu'à ces feuilles blanches qui tolèrent mon sillage, ou plutôt le sillage de ceux qui ont marché devant moi, pour moi. Je me suis avancé dans la trace de leurs pas comme dans un rêve éveillé où le parfum d'une pivoine éclose n'est plus une odeur, mais un épanouissement; $[\ldots]$ où un pays n'est plus un lieu, mais une berceuse.
\end{abstract}

Ces paroles reprennent le titre, elle revient à la signification de $r u$, pour clore ce ruisseau qui est la vie de la narratrice et de ses compatriotes exilés bien accueillis dans ce nouveau territoire. Elle n'est qu'une parmi eux pour poursuivre la transmission de la culture vietnamienne. Par l'écriture, elle montre sa gratitude au pays d'adoption et, surtout, à la langue française, celle qui lui a donné la connaissance du monde. Le Canada a donné l'occasion aux exilés de trouver une autre vie, il les a 'tolérés d'avancer parmi ces feuilles blanches', pas seulement à eux en tant qu'êtres humains mais à leurs ancêtres à travers leur culture et, de ce fait, les a légitimés pour intégrer ce bagage qui sert de 'berceuse' à leurs esprits, pour calmer leurs angoisses dans le pays d'adoption.

Le récit des gens et des personnes des deux pays qui ont composé la personnalité de la narratrice s'imprègne des sensations diverses, retrouvant chaque sens leur place dans les romans de Thúy. Dans celui-ci, par exemple, ce sont les odeurs autant de mauvaises que de bonnes, celles des lettrines dans le camp de réfugiés en Malaisie ou les parfums de violettes ou d'iris. Le moment où elle va recevoir son conjoint Guillaume à l'aéroport et celui-ci lui offre un flacon de son parfum préféré, réveille en elle tout un univers de sensations et de nostalgies parce que c'était celui qui imprégnait auparavant tous ses vêtements. L'odorat s'instaure en élément distinctif qui singularise une identité. À travers ce parfum, elle ressent pour la première fois le manque de son univers québécois et éprouve le 'mal du pays'. Dans le voyage à Paris, elle est nostalgique du passé québécois, mais elle sent aussi le besoin de repérer son parfum à elle pour l'avenir, celui capable de traduire les deux bouts d'une personnalité unique et unie, elle tente de retrouver une odeur qui lui donnerait "[mon] son pays, [mon] son univers" (Thúy, 2009: 117). La narratrice exprime le désir de trouver une fragrance différente capable de réunir son essence unique et complexe. Elle superpose des 
scènes et des points de vue qui tiennent souvent raison dans l'écart entre cultures; la fragmentation narrative du style de Kim Thúy répond à l'intérêt de donner corps à la matière composite des individus.

Les sens vont être atteints par les phrases qui évoquent le flair, mais le toucher est aussi convoqué, il sert à manifester l'attachement aux valeurs occidentales. An Tinh se fige sur des images chargées de poids documentaire de la distance culturelle, certains contacts physiques habituels pour les uns sont tout à fait inappropriés pour les autres. À l'instar, le geste de poser la main sur la tête d'un enfant devient habituel pour un Occidental mais il est considéré une insulte pour un Vietnamien (Thúy, 2009: 102). Elle montre comment la force des préjugés demeure dans chaque civilisation, la narratrice n'oublie pas de marquer l'importance du regard des autres sur celui qui n'est pas tout à fait conforme aux normes car l'exilé devient toujours l'autre. Elle éprouve ces préjugés personnellement car, en Orient, on ne la croit pas si elle dit qu'elle est vietnamienne parce qu'on la voit trop grosse malgré ses 45 kilos ou, au contraire, on ne la croit pas si elle dit qu'elle est canadienne en Occident à cause de ses yeux bridés.

Le temps intervient dans la conception de la réalité; elle voit les membres de sa famille si différents à ceux de leur arrivée qu'il est difficile de les reconnaître, le changement observé reste à l'origine de son style d'écriture. Elle note: "après seulement trente ans, je ne nous reconnais déjà plus que par fragments, par cicatrices, par lueurs" (Thúy, 2009: 141); la narration est tributaire de ces fragments et de ces cicatrices, elle ne montre pas la plaie mais la trace qu'elle a laissée et qui rayonne sur le présent. Pour Thúy, l'écriture est la manière de rescaper de l'oubli les êtres qu'ils ont été et qui ne seront plus.

Parmi les thèmes de cette première publication, nous retenons l'un d'eux qui nous semble s'éclairer en miroir avec la dernière publication de 2016: vi, celui du rapport mèrefille. Dans le roman de $r u$, la narratrice s'exprime ainsi para rapport à sa mère: "ma mère a commencé à vivre, à se laisser emporter, à se réinventer à cinquante-cinq ans" (Thúy, 2009: 72). Cependant, cette femme avait une forte personnalité et s'était instituée gestionnaire de l'autorité suprême face à ses frères et sœurs. La narratrice de $r u$ confesse vouloir devenir très différente de sa mère pendant son adolescence, mais cette distance envers la mère s'est estompée le jour où elle a pris conscience des valeurs à transmettre à ses enfants (Thúy, 2009: 59). An Tinh, en devenant mère à son tour, se réconcilie avec la figure maternelle puisqu'elle comprend, enfin, le rôle formateur qu'elle a joué dans sa vie infantile. L'union à la mère se décline de différentes manières dans les trois publications de notre étude. Comme souligne Ionescu (2014: 101): "La filiation lui a aussi permis de garder la mémoire vivante, de sauver de l'oubli des gens et des événements qui ont marqué son devenir personnel, intimement lié au devenir collectif'. À travers l'exercice de mémoire et d'écriture qui servent à mettre de l'ordre dans sa pensée, elle récupère l'autre bout de son identité qu'elle partage avec tant d'autres femmes des boat people. L'écriture de $r u$ est l'hommage à son héritage familial et la 
Anales de Filología Francesa, n. ${ }^{\circ}$ 27, 2019

Ángeles SÁNCHEZ HeRnÁNDEZ

réconciliation avec la figure maternelle que dans sa jeunesse n'avait pas été très fluide par les divergences culturelles qui piétinaient, à son tour, l'épanouissement de la mère.

\section{2. mãn}

Le titre du livre coïncide avec le prénom de la narratrice, Mãn, "qui veut dire 'parfaitement comblée' ou 'qui ne reste plus rien à désirer, ou que tous les vœux ont été exaucés" (Thúy, 2013: 34). D’après la tradition, son prénom était prémonitoire de son destin et, par conséquent, elle était destinée à un avenir sans rêves puisque ce nom signifie être complète et n'avoir besoin de rien de plus que ce qu'elle était et possédait. Mãn n'a jamais su qui était son père, mais son physique révélait qu'elle était la fille d'un “blanc, grand et colonisateur". Le récit se focalise sur celle qu'elle considère la vraie mère, la femme qui l'a élevée et accompagnée jusqu'à son mariage ${ }^{5}$. Dans la culture vietnamienne, l'amour ne se dit pas, ne se verbalise pas. Mãn dit: “de toutes les lettres que j'avais écrites à Maman, aucune ne contenaient ces trois mots 'Tu me manques', et pourtant chacun des détails racontés souffrait de son absence" (Thúy, 2013: 109). L'importance de la langue, du besoin de communication, est toujours présente; il faut nommer les choses, sinon elles semblent ne pas exister. Elle inscrit ainsi la gratitude à la figure maternelle, mais aussi la reconnaissance due à toutes les mères vietnamiennes qui ont vécu une expérience douloureuse à cause de la guerre ou l'exil. Toutes celles qui ont vu basculer leur vie "au son du premier tir d'une embuscade entre deux rives, entre l'Est et l'Ouest, entre la résistance qui réclamait l'indépendance et le régime en place qui enseignait aux élèves aux yeux bridés à dire 'nos ancêtres les Gaulois' sans y voir d'incohérence" (Thúy, 2013: 27). Elle remonte dans le temps pour évoquer la souffrance de la guerre, de l'invasion communiste mais, de plus, pour souligner le manque de respect du colonisateur par la culture de l'autre.

Chaque chapitre du livre porte un titre situé toujours dans la marge et écrit d'abord en vietnamien dans la graphie romanisée des Français, introduite pour les documents officiels, le Quốc-Ngữ, et ensuite elle note la traduction en français. Nathalie Edward (2018: 9) considère ce trait comme la trace d'un renversement ironique de la relation de pouvoir entre les deux langues; selon Edward, l'écrivaine développe une forme innovante d'auto-narration dans l'art de l'autoportrait avec cette manière de fusionner vietnamien et français vu que ce procédé fonde une dynamique d'utilisation des langues qui réussit à axer la narration essentiellement sur le langage. Mãn arrive au Canada après le mariage arrangé avec un Vietnamien qui s'était déjà fixé dans le pays sans arriver jamais à se sentir intégré. Les premières rencontres commencent sans presque aucun échange entre eux, perpétuant ainsi une tradition vietnamienne où peu de femmes se marient par amour. Le mariage organisé par les parents n'a pas

5 Elle en raconte trois: celle qui l'a donné la vie, une adolescente qui l'a déposée dans le potager d'un temple bouddhiste où une autre femme, sa deuxième mère, l'a allaitée un temps et, enfin, celle avec qui elle a vécu jusqu'à son mariage. 
besoin de manifestations affectives; inlassablement, le besoin de mettre en mots l'expérience pour lui donner de la visibilité (Thúy, 2013: 102):

Mon mari et moi n'avions pas adopté les baisers que les couples se donnent [...]. Nous
demeurions pudiques même après les deux enfants, même après vingt ans de mariage.
La langue nous contraignait à cette retenue. Nous parlions des choses en évitant de les
nommer. Il suffit d'être proche (gần) pour comprendre qu'il y avait eu relation sexuelle.
Il suffit que mon mari se tourne vers moi pour que je comprenne mon devoir d'épouse.

À son arrivée au Québec, Mãn travaille dans le restaurant géré par son époux s'appliquant alors à cuisiner selon les recettes traditionnelles que sa mère lui avait apprises. La cuisine est transmise de mère en fille presque à l'oreille pour protéger les recettes, pour qu'aucune saveur ne s'échappe et surtout pour garder le secret de ce qui fait alors le caractère unique de chaque plat; chaque mets raconte une histoire, un souvenir, ou est rattaché à un village. La jeune femme se lie d'amitié avec Julie qui devient sa collaboratrice dans le restaurant mais, peu à peu, elle devient 'sa grande sœur' et son initiatrice dans les mœurs occidentales; elle lui montre un autre comportement féminin dans une autre société. Son travail de restauration lui donne l'approbation générale de la clientèle et connaît ainsi un grand succès dans la ville, ce qui va lier son destin à celui d'autres personnages sortis de l'immigration. Comme on constate, l'écrivaine part encore une fois d'un fait de sa biographie pour développer l'intrigue du roman. L'attention portée à la cuisine demeure primordiale parce qu'elle se mêle au récit de la vie de sa mère, nous dévoilant l'univers intime de la narratrice à travers le dévouement à son métier. Dans l'histoire, s'entrelacent des amours différents, celui envers sa mère ou envers ses enfants et un autre encore à l'égard de Luc, réputé cuisinier français avec qui Mãn connaîtra la passion amoureuse. De plus, le personnage de Julie joue souvent le rôle de mère pour les enfants de Mãn; cette 'grande sœur' leur apprend d'autres valeurs éducatives de la culture occidentale qui permettent une communication plus fluide avec les enfants (Thúy, 2013: 108):

Dans une famille, 'Deux' exprime le premier rang et Julie occupait cette place puisqu'elle était plus âgée que moi, puisqu'elle était ma grande sœur. [..] Dès que Julie s'avançait pour les guider, les corriger, les amuser, je me retirais afin que la relation entre eux puisse s'approfondir et exister sans moi, après moi. Au Vietnam, il est dit que l'orphelin de père mange tout de même riz et poisson tandis que l'orphelin de mère doit étendre des feuilles par terre pour dormir $[\ldots]^{6}$.Mes enfants avaient beaucoup de chance. Ils avaient à la fois une assurance-vie et une assurance-mère.

L'influence de la tradition pèse lourd sur elle dans la façon d'élever ses enfants et elle trouve sa libération à travers la création culinaire. Dès le début, la narration est conduite par le sens du goût, "les mères enseignent à leurs filles à cuisiner à voix basse, en chuchotant, afin

6 Elle écrit à côté le proverbe en langue vietnamienne. 
d'éviter le vol de recettes par les voisines qui pourraient séduire leurs maris avec les mêmes plats" (Thúy, 2013: 12). Cette histoire d'amour filiale qui tient compte des enseignements maternels se relie à l'amour physique et fusionnel que Mãn découvre avec Luc, le cuisinier parisien. Cependant, cette situation amoureuse reste aussi dans l'inavouable car tous les deux sont mariés, une fois encore les mots ne suffissent pas. Alors, l'expression de la sensualité et de l'échange des amants va se faire par les images que génèrent les jouissances et les plaisirs du goût. Cette passion doit se taire car elle ne peut être vécue, c'est un amour commun né d'une rencontre ordinaire mais qui devient extraordinaire pour la narratrice, étant donné que c'est une expérience inattendue et inimaginable (Thúy, 2013: 122). C'est une initiation à l'écart de la tradition: "En moins d'une seconde son visage est apparu et, à cet instant précis, j'étais dans le présent; un présent sans passé". Tous les deux partageaient la profession et leur histoire d'amour se conjugue avec les mots de la cuisine, la sensualité du rapprochement des corps s'exprime par les procédés gastronomiques et à travers les perceptions gustatives.

La relation étroite entre cuisine et étreinte amoureuse a été l'objet d'une étude approfondie de la part d'Eileen Lohka (2017: 186). Dans son article sur cet aspect particulier de l'œuvre de Kim Thúy, elle souligne qu'il est facile de comprendre la proximité d'art culinaire et d'idylle développée entre Luc et Mãn si nous observons la place donnée aux plaisirs de la bouche, toujours reliés aux autres sens. En lisant les pages accordées à un événement culinaire où les deux amants travaillent ensemble dans la préparation des plats, le lecteur saisit l'envergure du trouble amoureux par la description des préliminaires pour faire la cuisine, l'étendue de la description occupe toute une page, tellement elle est minutieuse et caractérisée (Thúy, 2013: 128-129). De plus, en tant que restauratrice, Mãn retrouve la reconnaissance de son travail dans une profession dominée par les hommes, à l'encontre de la cuisine familiale communiquée presque à voix basse à la maison dans la lignée féminine. Elle raconte l'envie irrésistible de sortir de la ligne droite marquée par la tradition pour vivre une passion tumultueuse même si elle doit la cacher car cet acte est interdit par la tradition. Thúy sublime par son écriture poétique et sensuelle la vie routinière, fade et ordinaire, ainsi que le tourment mijoté dans son cœur.

\section{3. $v i$}

À nouveau, ce texte raconte l'adaptation d'une jeune fille à un nouveau pays où elle doit s'intégrer dans une culture éloignée de la sienne du fait qu'elle a été obligée de quitter son pays natal, le Vietnam. Elle perd ses racines pour se construire et se définir en tant que personne et, des années après, elle retourne au Vietnam chargée d'une mission professionnelle. Le livre s'ouvre sur la phrase de quelqu'un qui revient vers son enfance: 'J'avais huit ans quand la maison a été plongée dans le silence" (Thúy, 2016: 7). Elle va écrire à partir de ce silence, du moment de sa vie où il fallait taire toute nuisance pour pouvoir continuer 
à vivre. Le problème du silence et des non-dits apparait, à nouveau, comme pour réitérer et réaffirmer la nécessité de l'écriture, de mettre en lumière tous les silences vécus. L'acte d'écrire remplit une fonction existentielle chez Kim Thúy, mais elle sort de l'égocentrisme pour réaffirmer son engagement envers toute personne contrainte à l'exil sans la possibilité d'avoir une voix publique.

Les différents chapitres portent des titres qui nous emmènent en voyage du Mékong à Hanoi ou à Saigon, mais elle ne se limite pas aux pays reliés à son histoire biographique, Vi nous conduit autour du monde: Singapour, Kobé, Québec, Bruges, Manhattan ou Londres. Notre lecture nous conduit à l'intérieur des villes, elle nous mène par la rue Catinat dans l'ancien quartier chic de Saigon, plein d'hôtels luxueux ou de belles boutiques, ou bien nous faisons un grand bond qui nous transporte sur le chemin de la Côte-des-Neiges ou sur le boulevard René-Lévesque à Montréal, artère principale de la zone résidentielle de la ville avant de terminer ce tour au cœur de la colline parlementaire; elle le fait toujours en rapport direct avec l'événement de l'histoire qu'elle développe. Le récit s'imprègne des traits biographiques de l'auteure, elle démarre à l'âge de huit ans à Saigon au moment où la vie harmonieuse d'une grande famille vietnamienne est brisée par la guerre. La mère et ses enfants s'enfuient vers l'inconnu mais, dans ce cas, le père reste au pays et elle le retrouvera longtemps après à son retour au Vietnam. La narratrice Bao Vi dont le nom signifie 'précieuse minuscule' est, en revanche, grande et audacieuse; il y a un décalage important entre son physique et son prénom, symbole d'autres écarts de sa vie. Leur nouvelle existence est narrée par cette jeune fille tiraillée entre la fidélité aux traditions et ses propres aspirations de liberté. Le parcours de Vi ne se déploie qu'après une trentaine de pages consacrées aux ancêtres et à Hà, une autre exilée amie de sa mère qui aura sur Vi une influence libératrice. La jeune fille a le courage de surmonter les préjugés que la tradition familiale lui imposait pour imposer son choix d'études supérieures, contredisant la volonté parentale.

L'auteure essaie d'établir le passage d'une génération à l'autre de faire le point sur ce qu'il faut retenir et ce qu'il faut laisser derrière soi, mesurant bien l'influence de la tradition dans une vie. Kim Thúy avoue que ses héroïnes sont des fantasmes d'elle-même et, à travers ses personnages, elle rend visible beaucoup de ce qu'elle aimerait être ${ }^{7}$. $V i$ raconte le parcours de construction identitaire d'une femme destinée à rester invisible dans la tradition vietnamienne. Elle grandit dans une société avec d'autres valeurs mais elle n'arrive pas à se libérer ni de son prénom et ni de ce qu'il colporte. Bao Vi évolue dans sa vie doucement, sa personnalité se forme à travers ses rencontres et ses déplacements où tous les détails s'imbriquent composant de brefs tableaux, chacun représente un fragment de l'histoire, mais la superposition des uns et des autres compose le tableau complet de la vie du personnage et de son entourage. Le récit ne peut pas se dissocier de la structure narrative qui l'entraîne (González Menéndez, 2014: 185).

7 Vidéo de présentation de Kim Thúy pour la librairie Mollat de Bordeaux, disponible sur $<$ https://www.youtube. com/watch?v=uvy58IZQ5Jo $>$. 
Kim Thúy recherche la précision linguistique, souci hérité de la profession d'avocate; en revanche, elle croit que la patience pour poursuivre le procès nécessaire pour écrire dérive du métier de cuisinière. Le désir de clarté et de justesse linguistique est lié à la nécessité de transposer, par le choix des mots, la diversité des impressions sensitives. Son expérience de mère d'un enfant autiste reste à l'origine du besoin de communiquer, mais elle traduit cette exploration sensorielle en mots. Elle cherche à inciter le monde sensible du lecteur; surtout, à travers la place des mots dans le récit pour aboutir à l'échafaudage final du texte qui, malgré ces 'bulles de mémoire' dont parle Thúy, reste cohérent et connexe. L'écriture fragmentée et la recherche du mot précis délivrent une sensualité multiple qui manifeste l'importance essentielle de la relation entre le corps et l'espace pour cette écrivaine qui rédige "des passages poétiques traitant du sentir et de la perception" (Sing, 2013: 283). L'impression sensorielle est manifestée de manière subtile et délicate pour entourer le lecteur d'une légèreté joyeuse, la narration s'écarte du récit dramatique des événements sans négliger la dureté des faits, fouillant patiemment dans les impressions pour percer dans la nuance capable de rendre la sensation ressentie. Cette recherche stylistique naît de sa relation à la langue vietnamienne, restée selon Thúy au niveau de langue enfantine, car elle est capable de 'ressentir le livre' qui est écrit en cette langue si les images dénotées par les mots sont capables de faire ressortir en elle la sensation visée; elle tente de reproduire le même effet sur ses lecteurs.

Nous avons parlé de la relation mère-fille poursuivie de manières diverses dans les romans analysés. Dans cette dernière publication, les aspects soulignés sont surtout ceux dans lesquels la jeune fille s'écarte de la tradition et cherche sa voie personnelle qui est imprégnée de culture occidentale. Elle cherche son chemin et cette quête identitaire la mène à se débarrasser des normes, particulièrement en ce qui concerne le choix de mari. Fiancée à Tân, un étudiant vietnamien de la diaspora comme elle, mais aux idées assez conservatrices qui lui font rejeter sa petite amie pour s'être donné sexuellement à lui avant le mariage comme Vi l'avait fait, alors ils ne célèbrent les fiançailles que pour sauvegarder les convenances. Tân n'approuve aucun comportement de sa future femme et, à nouveau, le langage de la cuisine se lie à un fait grave; dans ce cas, il signale la rupture amoureuse (Thúy, 2016: 95). Elle note: “à partir de cette nuit d'euphorie entre jeunes qui avaient pour philosophie work hard, play harder, Tãn ne s'est plus contenté des ragoûts que je confectionnais". Vi suit alors la consigne de Hà qui avait insisté pour qu'elle ne se marie pas avant l'âge de trente ans et la jeune fille a envie d'aller ailleurs, envie de voyage physique mais aussi de voyage intérieur.

Hà lui a fait découvrir le monde, elle l'avait emmenée à Manhattan à 13 ans et lui a donné le pouvoir de choisir, de sortir du cercle d'influence de la famille. Son frère Long reprochait à la mère de l'avoir confiée à Hà, changeant ainsi un parcours qui devait être stable et facile pour un autre imprévisible et chaotique (Thúy, 2016: 69). Le modèle féminin qu'incarne cette femme joue le rôle de personnage initiateur vers un monde où la sexualité est vécue différemment de ce que voudrait sa vraie mère. Et cependant la mère l'a confiée 
à Hà, sachant l'effet qu'elle produirait sur sa fille, mais elle était incapable de lui donner la liberté qu'elle s'interdisait à elle-même. À 15 ans, Vi se rebelle contre la conception de virginité que sa mère lui conseillait de préserver; l'adolescent lui répond ainsi: “un corps n'est pas une chose. Il ne peut donc être neuf ou usé ou usagé” (Thúy, 2016:78). La mère est assez lucide pour comprendre que cette pensée provenait des idées de Hà ainsi que de la lecture d'articles dans les magazines sur les viols en mer vécus par des boat people ou de la lecture des romans comme ceux de Marguerite Duras qu'elle admirait. La figure maternelle retrouve une ambivalence dans le roman car Hà a pu entrer dans l'existence de Vi par sa permission, elle était tiraillée entre la propre éducation dans la tradition et la compréhension du besoin d'un changement. La mère était consciente qu'elle ne pouvait pas éduquer sa fille dans les mêmes valeurs qu'elle avait reçues puisque la jeune fille appartenait à un monde distinct des origines vietnamiennes; elle s'était rendu compte du besoin d'un développement plus libre et émancipé des femmes.

\section{Conclusion}

Kim Thúy passe dans son œuvre du moi privé au moi social et s'intéresse aux composantes changeantes et insaisissables de l'identité qui est bâtie autant sur le présent que sur le passé. En écrivant, elle est obligée de descendre vers ce moi inexploité qui semblait égaré dans le passé pour composer le personnage qui parle à la première personne. Comme notait Paul Nizon, ce ‘je' n'est donc pas le point de départ de l'autobiographie, mais le point d'arrivée car il devient un être composite des fragments —ou 'bulles' comme propose Kim Thúy_ du passé vécus par ce 'je'. Il ne s'agit plus de rester fidèle aux faits et au temps du moi, mais de se rapprocher de ce qui a survécu dans la partie la plus intime de l'être qu'elle est aujourd'hui et qui est perçu comme élément formateur de la personnalité qui l'habite.

L'auteure met en ordre le vécu, la vie au quotidien d'une des boat people qui tente de saisir la signification de la perte du pays, comprenant enfin que l'exil est devenu chez elle une addition humaine et culturelle et non pas une soustraction. Elle s'est nourrie de cette expérience et la perçoit comme une opportunité de s'ouvrir au monde pour s'épanouir, ce point de vue donne le ton joyeux à ses récits. Elle observe ces jeunes femmes qui sont ses personnages comme des facéties d'un seul individu, elle-même. Dans un entretien télévisé, l'auteure a reconnu que Vi est la femme qu'elle voudrait être ; cette femme qui, partant de cette petite fille qui fut An Tinh en ru, a évolué au fil du temps sur le territoire d'adoption pour s'accomplir en tant que femme. Elle a voyagé à travers le monde et les cultures pour trouver sa place dans la société et se retrouve femme adulte ayant su trouver son parcours individuel librement sans oublier ses racines. L'hommage à la mère, aux différents types de mères représentées dans les romans, malgré la révolte de sa jeunesse contre une tradition que 
la contraignait et voulait la forcer à la soumission; ces mères sont les gardiennes des valeurs traditionnelles sachant toujours s'adapter aux nouveautés.

Son écriture est singulière et personnelle par cette fragmentation narrative qui sait bien s'agencer pour composer le tableau biographique. Elle dépasse le réel pour donner à entendre les révoltes, les quêtes intimes et le bonheur expérimentés qui sous-tendent l'existence. Elle s'éloigne sans doute de l'autobiographie 'normative' pour gagner la possibilité de dénuder l'épaisseur de l'expérience humaine ; dans son cas, l'expérience de l'être exilé. Chez Kim Thúy, l'écriture répond à une motivation rationnelle essayant d'avancer du 'je' au 'nous', de même qu'à une motivation affective par le plaisir qu'elle éprouve en travaillant les mots; ses récits remplissent une fonction gnoséologique remettant de l'ordre dans les événements qui ont composé son identité.

\section{Références Bibliographiques}

Blanckeman, Bruno. 2002. Les fictions singulières. Paris, Prétexte éditeur.

BRUnel, Pierre. 2008. "Introduction aux autobiographies" in Revue de littérature comparée, $\mathrm{n}^{\mathrm{o}} 325,7-22$.

Cuasante Fernández, Elena. 2018. "Las escrituras del yo y sus variantes funcionales" in Revista de Filología, no 37, 25-39.

Chaudey, Marie. 2010. "Kim Thúy raconte sa renaissance" in La Vie, no 3361, [consulté le 12/03/2019] <http://www.lavie.fr/hebdo/2010/3361/kim-thuy-raconte-sa-renaissance-26-01-2010-2410_97.php>

Dusaillant Fernandes, Valérie. 2012. "Habiller le vécu de mots et d'images: le projet de Kim Thúy", in Voix plurielles, 9 n² 2, 164-177.

EdWARDS, Nathalie. 2018. "Linguistic Rencontres in Kim Thúy’s Mãn”, in Journal of Multidisciplinary International Studies, vol. 15, nº1-2, 6-19.

Fortin, Marie-Claude. 2009. "Ru de Kim Thúy. À fleur de peau” in La Presse. [consulté le 22/03/2019] <https://www.lapresse.ca/arts/livres/200911/27/01-925704-ru-de-kim-thuya-fleur-de-peau.php $>$.

Gasparini, Philippe. 2011. “Autofiction vs autobiographie" in Tangence, $\mathrm{n}^{\circ}$ 97, 11-24.

Grell, Isabelle. 2014. L'autofiction. Paris, Armand Colin.

Grell, Isabelle. 2016. "Il est temps de réagir" in Les enjeux (en-je) de la chair dans l'écriture autofictionnelle. Louvain-La-Neuve, EME éditions, coll. "Proximités", 5-10.

GonzÁLez MenÉndez, Lidia. 2014. "Lengua, escritura y arraigo en la obra de Kim Thúy”, in Çedille 10, 181-192.

GouIfFès, Nathalie. 2002. Le biographique. Paris, Magnard. 
IonEsCU, Mariana. 2014. "L'écriture de Kim Thúy et Liliana Lazar: résilience ou résistance?", in@nalyses. Revue de critique et de théorie littéraire, vol. 9-nº 3, 95-111.

LALONDE, Johanne. 2018. Le fragment dans l'autoportrait littéraire, in @analyses. Revue de critique et de théorie littéraire, vol. 13-nº 1, 32-55.

Lejeune, Philippe. 1975. Le pacte autobiographique. Paris, Éditions du Seuil.

Lejeune, Philippe. 2010. L'autobiographie en France. Paris, Armand Colin.

Le Quellec Cottier, Christine. 2012. "Cendras et ses écrits autobiographiques: une autofiction avant la lettre?" in ZuFFEREY, Joël (éd.) L'autofiction: variation génériques et discursives. Louvaine-La-Neuve, L'Harmattan-Academia.

LoHKA, Eileen. 2017. "Senteurs de l'ailleurs: Mémoire culinaire chez Kim Thuy" in Nouvelles Etudes Francophones, vol. 32- nº 2, 184-194.

NAnCy, Dominique. 2013. "Kim Thúy, l'auteure assouvie" in Journal FORUM, [consulté le 12/04/2019] <http://www.nouvelles.umontreal.ca/culture/livres/20130415-kim-thuylauteure-assouvie.html $>$.

Nizon, Paul. 2005. La République Nizon. Rencontre avec Philippe Derivière. Paris, Éditions d'Argol, coll. "Les Singuliers".

Romera Pintor, Ángela Magdalena. 2018. "Fundamentos teóricos y críticos de la escritura autobiográfica femenina en lengua francesa” in Revista Signa, n 27, 17-37.

Sing, Pamela V. 2013. "Migrance, sesorium et trasculturalité chez Ying Chen et Kim Thúy", International Journal of Francophone Studies, 16-3, 281-301.

Simon, Geneviève. 2017. "Foire du libre: Revivez la conférence 'Quand tout a basculé'avec Gaël Faye et Kim Thúy” in La Libre Belgique, vidéo en ligne [consulté le 12/04/2019]<https:// www.lalibre.be/culture/livres-bd/foire-du-livre-revivez-la-conference-quand-tout-a-basculeavec-gael-faye-et-kim-thuy-video-58c510f2cd705cd98de59e95>

Tavana, Elham, Zohreh Nassehi, \& Jamshid A. Azghandi. 2013. "Autofiction doubrovskienne ou fiction au service de la vérité autobiographie" in Recherches en Langue et Littérature Françaises. Revue de la faculté des Lettres, vol. 7-12, 123-142.

ThÉriault, Mélissa. 2016. “Ce corps qui n’est pas soi: nihilisme, dualisme et autofiction chez Arcan" in Les enjeux (en-je) de la chair dans l'écriture autofictionnelle. Louvain-LaNeuve, EME éditions (coll. Proximités), 27-41.

THúY, Kim, 2009, ru. Paris, Éditions Liliana Levi.

ThúY, Kim, 2013, mãn. Paris, Éditions Liliana Levi.

ThúY, Kim, 2016, vi. Paris, Éditions Liliana Levi.

ThúY, Kim \& Gaël FAYE. 2017. "Quand tout a bousculé”, in La Libre.be. [consulté le 11/04/2019]<https://www.lalibre.be/culture/livres-bd/foire-du-livre-revivez-la-conferencequand-tout-a-bascule-avec-gael-faye-et-kim-thuy-video-58c510f2cd705cd98de59e95>

Zanone, Damien. 1996. L'autobiographie. Paris, Ellipses. 